\title{
SUPERSONIC TESTING OF NAL BINARY PRESSURE SENSITIVE
} PAINT

\author{
Sathia Narayanan*, Lakshmi, R.V ${ }^{+}$, Bharathibai J. Basu ${ }^{+}$, Channa Raju* and Venkatakrishnan, L ${ }^{*}$
} Council of Scientific and Industrial Research, National Aerospace Laboratories, Bangalore, India "Experimental Aerodynamics Division, ${ }^{+}$Surface Engineering Division

\begin{abstract}
The main objective of the present study is to subject NALPSP-02, an indigenously developed binary pressure sensitive paint, to supersonic flow conditions. An experimental study was carried out on a double-delta wing model at a free stream Mach number of 2.0 in the $0.3 \mathrm{~m}$ trisonic wind tunnel at NAL. Pressure Sensitive Paint (PSP) technique was used to measure the pressure distribution on the lee side of the double delta wing at an incidences of $0^{\circ}, 5^{\circ}, 10^{\circ}$ and $15^{\circ}$. The PSP results were compared against surface pressure data obtained using conventional pressure taps on the same model.
\end{abstract}

Key Words: Pressure sensitive paint, Delta wing, supersonic flow

\section{INTRODUCTION}

Delta wings have advantages over conventional aerofoils in terms of aerodynamic characteristics. The counter rotating leading edge vortex pair creates high suction on the top surface of the wing. This energised vortex pair delaying the separation stall and thus leads to higher stall angles. Research on flows over delta wings has tremendous effect on the design and development of high speed fighter aircrafts and missiles [1,2].

To measure the surface pressure distribution on any wind tunnel model, conventional pressure measurement systems which are time consuming and limited in spatial resolution are being used. Alternative to this measurement is the pressure sensitive paint (PSP) technique which is based on the principle of oxygen quenching of luminescence [3]. PSP offers higher spatial resolution compared to the conventional electronic sensor based pressure measurement systems.

An intensity based PSP system is being used in the NAL $0.3 \mathrm{~m}$ blow down tunnel $[4,5]$. The paint sensor is a key element of the system. Earlier PSP efforts at NAL used the commercially available Optrod-B1 paint. Efforts have been underway to develop an indigenous industry standard binary PSP and the processing software [6 -9]. Calibration tests and the wind tunnel aging tests in the $0.3 \mathrm{~m}$ tunnel have confirmed that paint formulation NALPSP-02 is on the advanced stage of development and is ready for tunnel applications in transonic and supersonic flow. This study on a delta wing is to determine the applicability of the PSP so developed.

\section{EXPERIMENTAL SETUP}

The experiments were conducted in the NAL $0.3 \mathrm{~m}$ trisonic blow down test facility which has a square test section of $0.3 \mathrm{~m} \times 0.3 \mathrm{~m}$ [Figure .1]. The tunnel can provide Mach numbers ranging from 0.2 to 4.0 and a Reynolds number range of 15 million to 60 million per meter. Total pressure for the current experiments was set at an absolute pressure of $206 \mathrm{KPa}$ which resulted in a unit Reynolds number of 44 million. All tests were carried out at a tunnel total temperature of $303^{\circ} \mathrm{K}$.

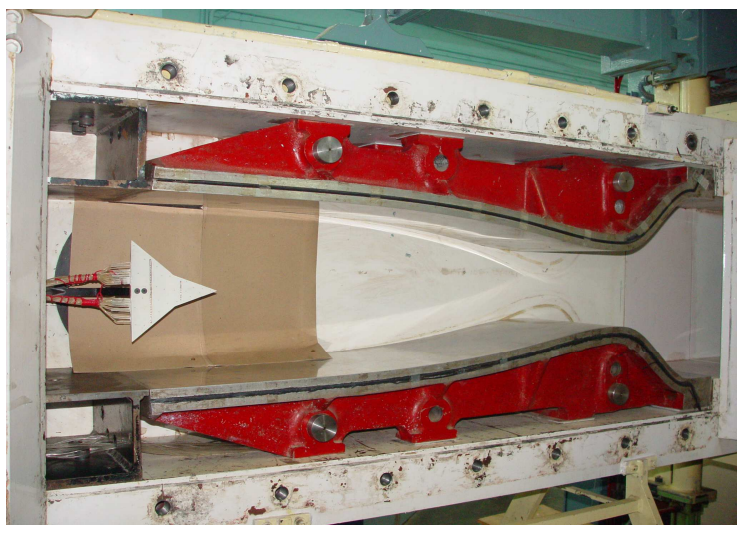

Figure 1. Photograph of model mounted in the 0.3 m Trisonic Tunnel

\section{MODEL DETAILS}

Figure 1 shows the model mounted in the $0.3 \mathrm{~m}$ trisonic wind tunnel. The dimensions of the model used in the study are given in Figure 2. The model is a double delta wing configuration without the fuselage section. The model has a first sweep angle of $69^{\circ}$ and $47^{\circ}$ as the second sweep angle. The length (l) and span (b) of the model are $148 \mathrm{~mm}$ and $180 \mathrm{~mm}$ respectively. The model was mounted on a bent sting to attain the required angle of attack $(\alpha)$. Three bent stings were used to vary the angle of attack. The model was fixed with the lee ward side facing the modified Schlieren window in the tunnel to facilitate imaging. 


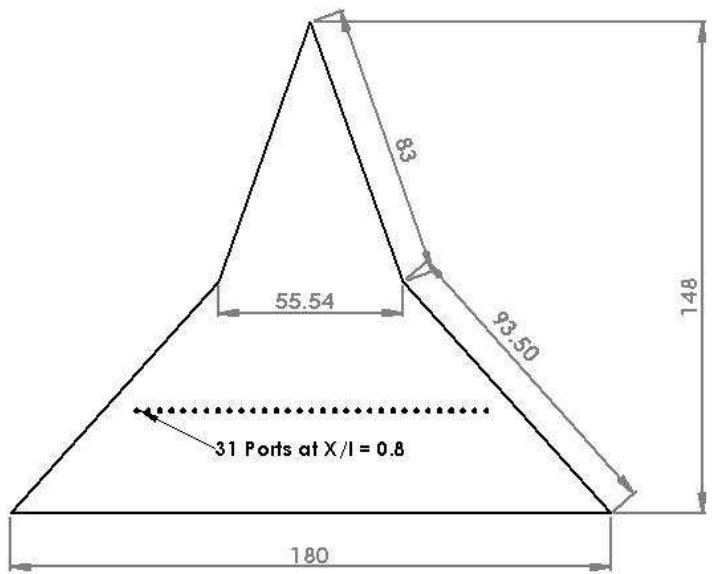

Figure 2. Dimensions of the Model in mm

The maximum blockage of $8 \%$ occurred at $15^{\circ}$ angle of attack. The model was provided with 31 static pressure ports of $0.5 \mathrm{~mm}$ dia to validate the PSP measurement. Two differential ESP scanners of range $\pm 103 \mathrm{Kpa}$ were used to measure the surface pressure distribution through the ports laid on the model. The uncertainty estimated was in the range of $\Delta \mathrm{Cp}< \pm 0.02 \mathrm{Cp}$.

\section{PSP SETUP}

A modified Schlieren window on the side wall of the test section provided the optical access for illumination of the model as well as detection of emission from the model [Figure 3]. The model was illuminated using a Xenon flash lamp providing UV excitation of the paint in the range of $300 \mathrm{~nm}$. The pressure sensitive and intensity images were captured using two identical peltier cooled 12-bit scientific grade CCD cameras sensitive to the the ranges of $450-550 \mathrm{~nm}$ and 600-650 $\mathrm{nm}$ respectively.

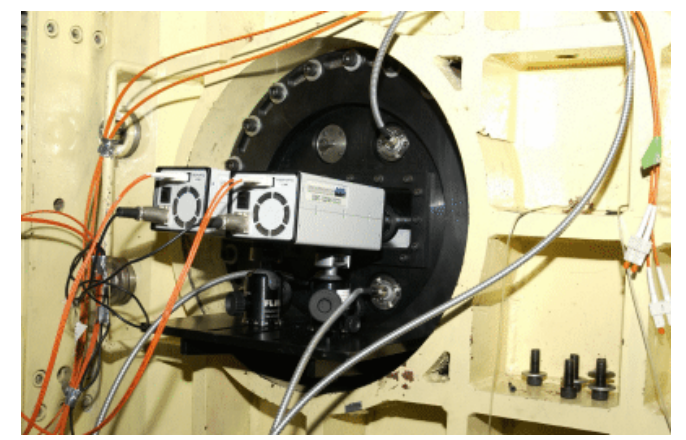

Figure 3. PSP arrangement and Optical Access

The intensity images were converted to the corresponding pressure images based on a priori coupon calibration obtained in a special calibration chamber. The temperature coefficient of the NALPSP-02 paint is small $\left(<0.3 \% /{ }^{\circ} \mathrm{C}\right)$, and hence the PSP data does not require any temperature corrections [9].

\section{CALIBRATION}

Prior to the wind tunnel experiments, Aluminum coupon of $25 \mathrm{~mm}$ by $25 \mathrm{~mm}$ in size coated with NALPSP-02 was calibrated. The pressure in the calibration chamber was varied from $100 \mathrm{mbar}$ to 1600 mbar and the corresponding pressure sensitive and illumination reference images (at a temperature of $25^{\circ} \mathrm{C}$ ) were acquired. The variation of intensity in sensitive, reference and normalized images with respect to pressure is shown in Figure 4. The normalising pressure was taken to be 1 bar and the sensitive and reference images corresponding to this pressure were taken as normalising images. The results from the calibration data was used to process the images acquired during wind tunnel experiments. The pressure sensitivity of paint was $64.57 \%$ / bar.

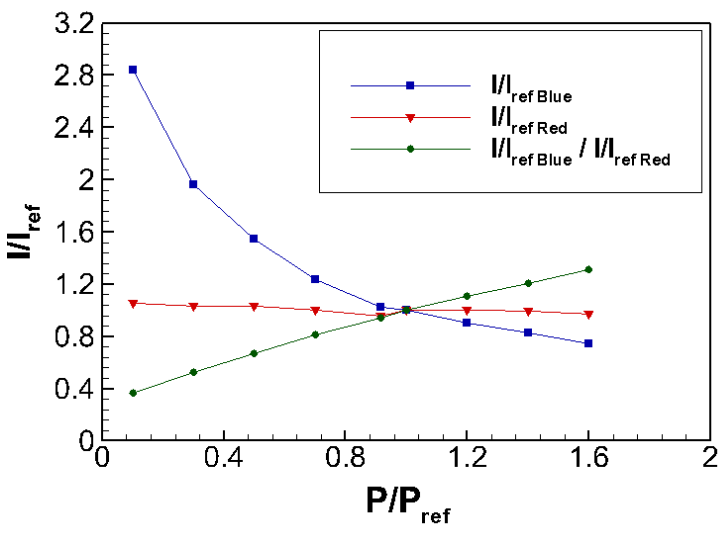

Figure 4. Variation of intensity with pressure

\section{PSP DATA PROCESSING}

In house developed Matlab based software [6] was used to process the PSP images. The software uses the resection based approach, which incorporates the collinearity equations of photogrammetry. A comprehensive camera model, which takes into account the lens distortions as well as errors in identifying marker locations, is used to resect each of the wind-on and wind-off images from both cameras onto a 3D body surface grid, prior to ratioing. A total number of twenty seven markers were used as control points during the image processing. These points are visible in the PSP images shown later. 


\section{RESULTS AND DISCUSSION}

7.a $\alpha=0^{\circ}$

Figure 5 shows the $\mathrm{Cp}$ distribution on the delta wing at an angle of attack of $0^{\circ}$. It is seen that the pressure map is highly informative and has completely captured the pressure distribution over the entire surface. It may also be noted that the PSP image is not just a flow visualisation; alternately it gives quantitative data of surface pressures at every location on the model surface. The two red circles seen are the model mounting locations. The surface pressure ports were covered using adhesive tape during model coating and is seen as a horizontal red stripes across the model at two locations. The higher $\mathrm{Cp}$ shown at the port side edge of the main wing is due to the error in mapping. A very weak vortex originating from the strake is captured by PSP as a reduction in pressure is seen in this region.

Comparison between PSP and ESP measurements at $0^{\circ}$ is shown in Figure 6. PSP measurement matches well with the ESP measurement. The model exhibited a slight roll $\left(<1^{\circ}\right)$ during blowdown. This causes an asymmetric flow development on the model and is captured in the corresponding span wise $\mathrm{Cp}$ distribution. Both the primary and secondary vortices were captured well by the PSP measurement.

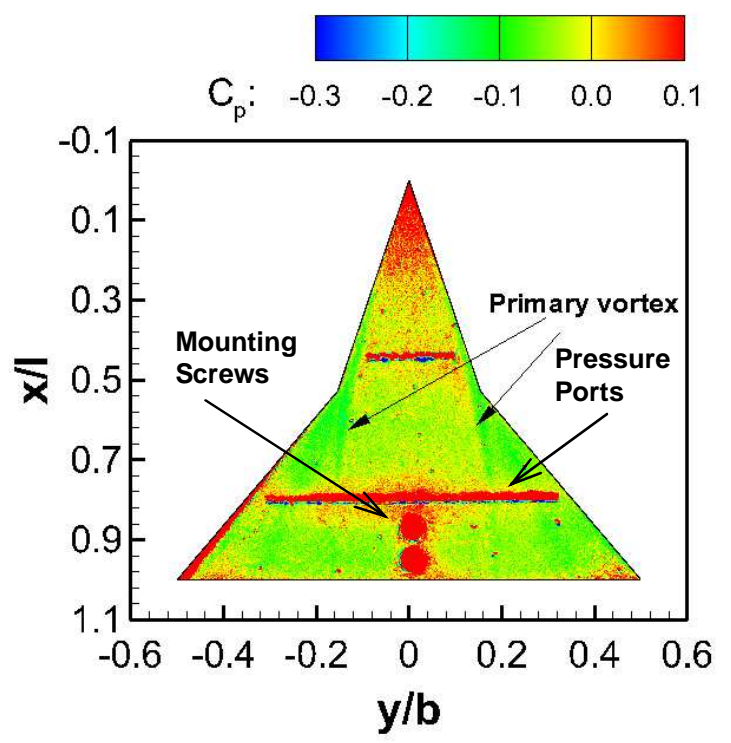

Figure 5. Cp distribution over delta wing at $0^{\circ}$

The surface flow pattern visualized using surface oil flow visualisation is shown in Figure 7. A weak vortex from the strake leading edge is clearly seen. The asymmetric flow captured in PSP and ESP measurement is also visible in the picture shown.

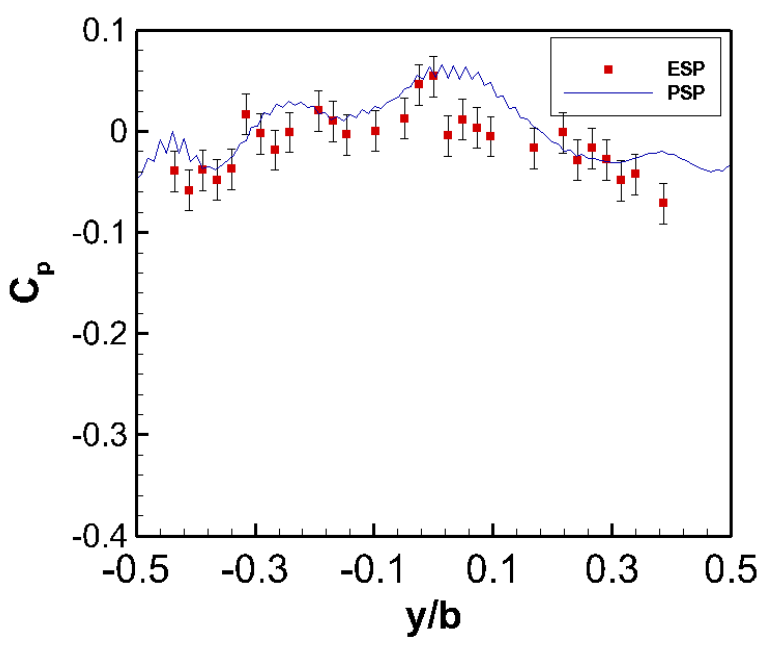

Figure 6. Comparison between ESP and PSP measurement at $0^{\circ}$

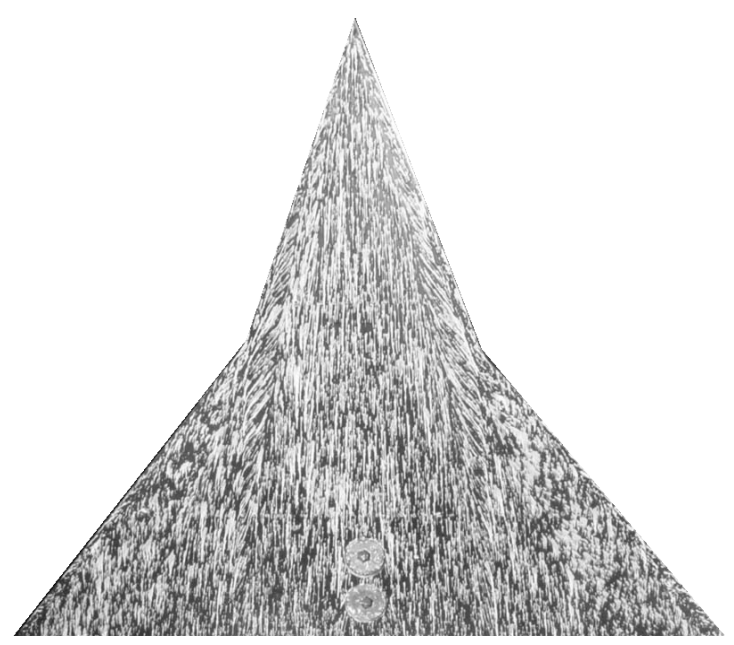

Figure 7. Surface flow pattern at $0^{\circ}$

7.b $\alpha=5^{\circ}$

Pressure field captured by PSP, comparison between PSP and ESP and the surface flow pattern at $5^{\circ}$ are shown in Figures 8 to 10 . The dominating primary vortex from the strake leading edge is clearly visible in the PSP field. The pressure difference occurred due to the presence of the counter rotating vortex pair was captured well by PSP measurement.

Comparison of $\mathrm{Cp}$ measured by PSP against ESP measurement revealed that PSP captured the Cp distribution across the primary and secondary vortex. The reduction in $\mathrm{Cp}$ across vortices is larger than that of at $0^{\circ}$. 
Surface oil flow pattern shows that the flow is almost symmetric about the stream wise axis. The vortex from the main wing does not contribute much to the flow and a dead air region is observed near the trailing edges.

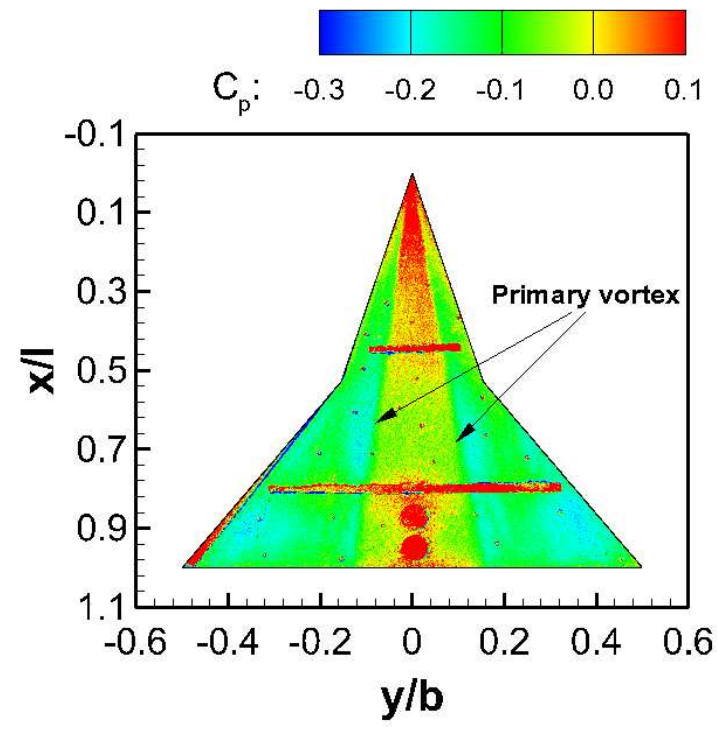

Figure 8. Cp distribution over delta wing at $5^{\circ}$

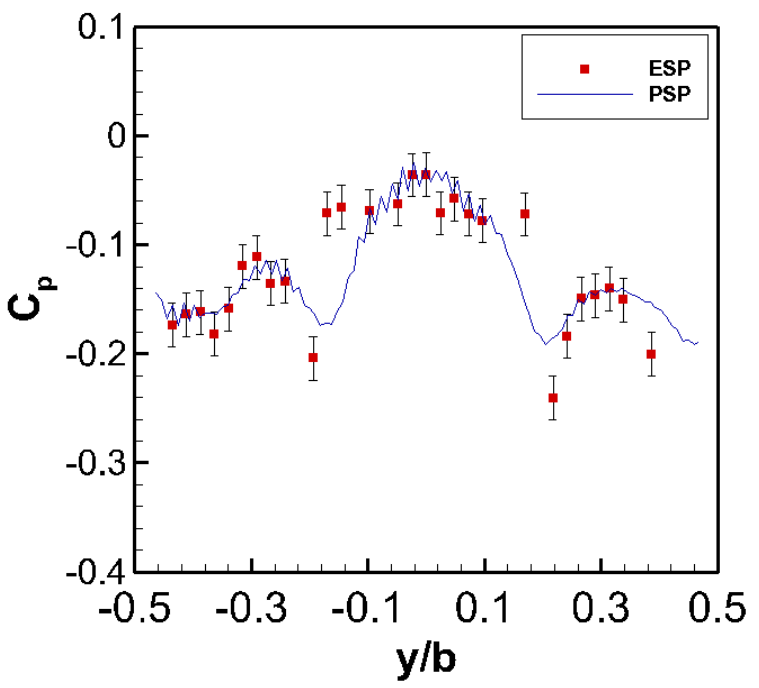

Figure 9. Comparison between ESP and PSP measurement at $5^{\circ}$

7.c $\alpha=10^{\circ}$

Cp distribution at $10^{\circ}$ is shown in Figure 11. The comparison between ESP and PSP measurement is shown in Figure 12. PSP data matches very well with the ESP data. Surface flow pattern shown in Figure 13 shows the vortex induced span wise flow in the center portion of the model.

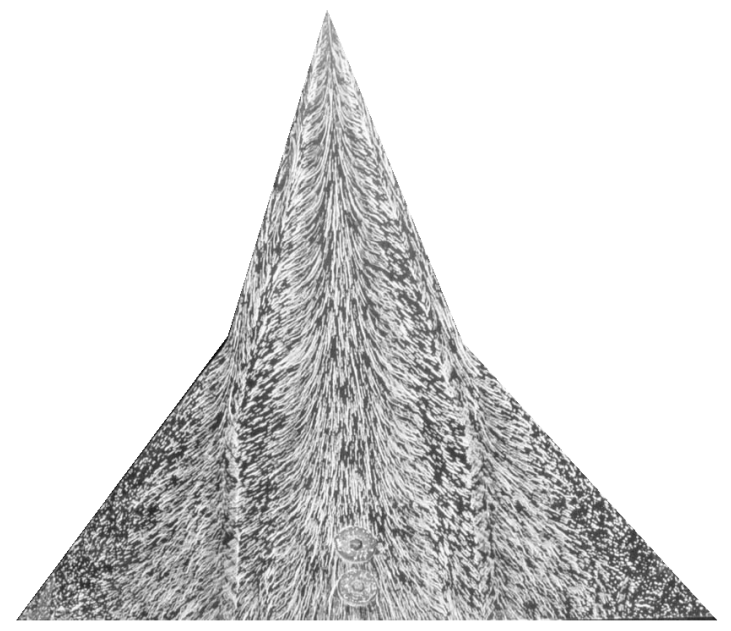

Figure 10. Surface flow pattern at $5^{\circ}$

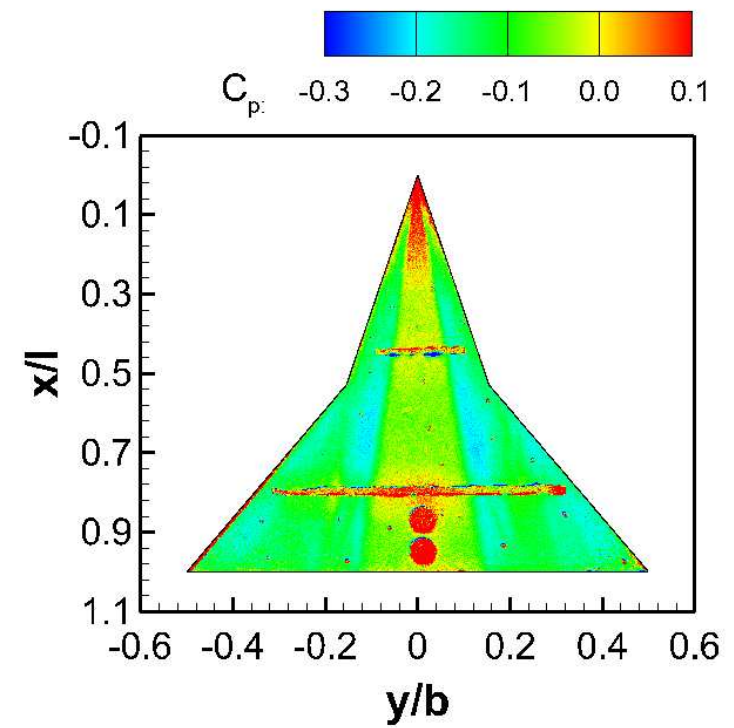

Figure 11. Cp distribution over delta wing at $10^{\circ}$

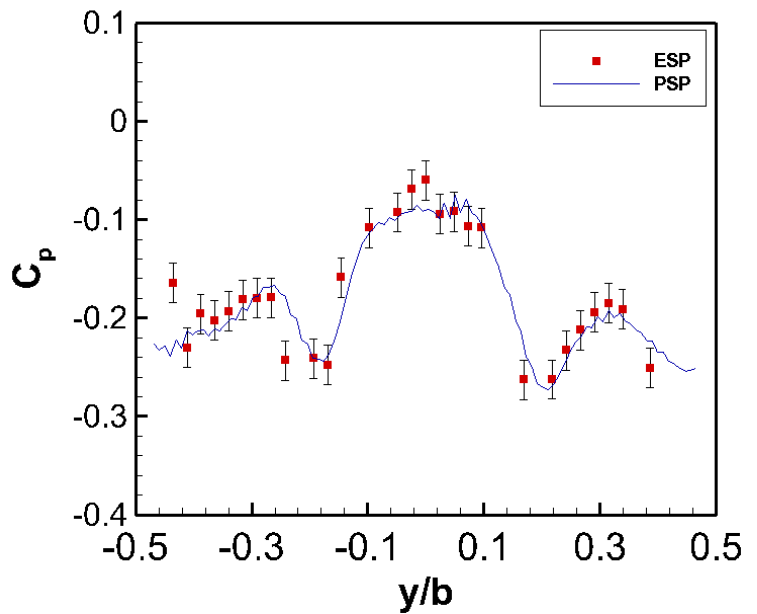

Figure 12. Comparison between ESP and PSP measurement at $10^{\circ}$ 


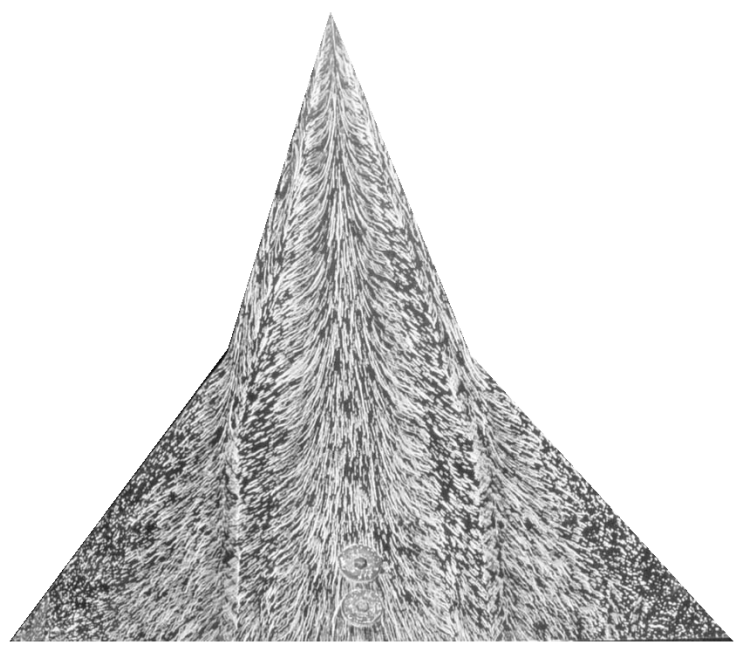

Figure 13. Surface flow pattern at $10^{\circ}$

7.d $\alpha=15^{\circ}$

Figure 14 shows the $\mathrm{Cp}$ distribution captured by PSP at $15^{\circ}$. Comparison of Cp captured by PSP against the Cp measured by ESP is shown in Figure 15. The reduction in $\mathrm{Cp}$ across the primary vortex is greater than the reduction in $\mathrm{Cp}$ at lower angles of attack since the strength of the vortex increases with increase in angle of attack. Surface oil flow pattern is shown in Figure 16. The size of the primary vortex from strake leading edge is increasing with angle of attack. Secondary vortex on the star board side of the strake is clearly visible.
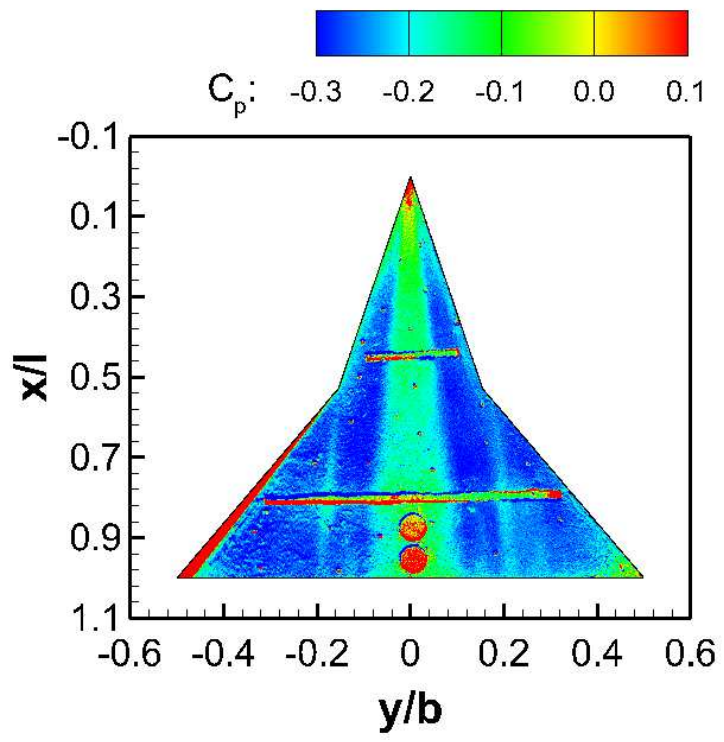

Figure 14. Cp distribution over delta wing at $15^{\circ}$

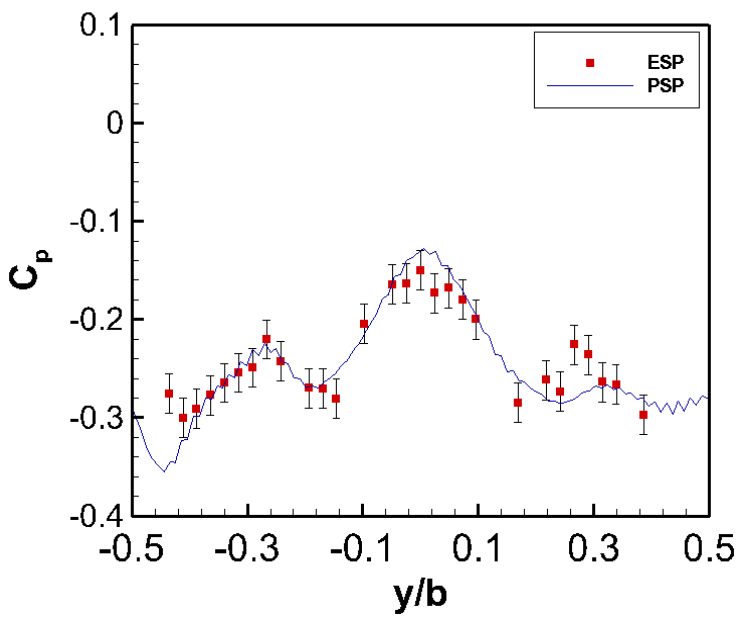

Figure 15. Comparison between ESP and PSP measurement at $15^{\circ}$

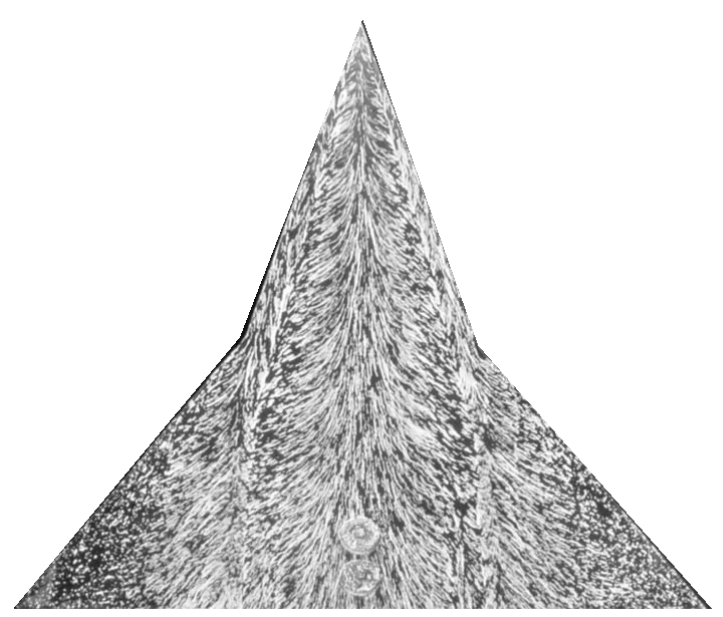

Figure 16. Surface flow pattern at $15^{\circ}$

\section{CONCLUSIONS}

Wind tunnel experiments have been carried out over double delta wing coated with NALPSP-02. The results showed a satisfactory performance of NALPSP-02 in supersonic regime. The surface pressures as obtained from PSP match that from conventional measurements to within $6 \%$. Thus it is concluded that NALPSP-02 can be applied to supersonic pressure measurements in blow down wind tunnels.

\section{ACKNOWLEDGEMENTS}

Authors thank Mr. Periya Nayagam and Mr. Muthusamy for their assistance during the wind tunnel experiments and Mr. Janardanan for his help during the data acquisition and Mr. Dinesh Kumar for the help in coating the model with NALPSP-02 paint. 


\section{REFERENCES}

1. Terence, A., Hugo, A. and David, B. : Experimental investigation of vortex-tail interaction on a 76/40 degree double delta wing, AIAA 993159

2. William, H. and Michael, C. : An experimental investigation of the flow fields about delta and double delta wings at low speeds, NASA CR 521

3. Liu, Tianshu and Sullivan, J : Pressure and Temperature Sensitive Paints, Springer, 2004

4. Channa Raju and Viswanath, P.R : PSP measurements on an aircraft model in a blowdown wind tunnel, Journal of Aircraft, 2005, 42(4), 908-915.

5. Channa Raju, Venkatakrishnan, 1 and Viswanath, P.R : "PSP measurements on a delta wing in supersonic flow", The Aeronautical Journal of the Royal Aeronautical Soc., Nov. 2006

6. Venkatakrishnan, L. "A comparative Study of Different PSP image Registration Techniques", 2004, AIAA J, 42 (11), 23112319.

7. Bharathibai J. Basu, NAL Binary PSP: Development of new paint formulations, NAL PD SE 0509, June 2005.

8. Channa Raju, S.B. Verma, Bharathibai J. Basu and S. Venkatraman, NAL Binary PSP: Aging Tests at transonic speeds - Part IV, NAL PD EA 0510, August 2005.

9. Bharathibai J. Basu, Vasantharajan, N and Channa Raju, A novel pyrene-based binary pressure sensitive paint with low temperature coefficient and improved stability, Sensors and Actuators B 2009, 138, 283-288. 